\title{
Benign Paroxysmal Positional Vertigo: Our Experience
}

\author{
K. K. Patangay $\cdot$ Rahbar Ansari
}

Received: 3 November 2014/ Accepted: 9 December 2014/Published online: 16 December 2014

(C) Association of Otolaryngologists of India 2014

\begin{abstract}
Benign paroxysmal positional vertigo (BPPV) is probably common cause of vertigo. A total of 205 cases reported to ENT OPD for vertigo. Of these 43 patients were found to suffer from BPPV and in our experience BPPV is common condition. BPPV was more common in age group of 4th and 7th decades. The youngest patient reported was 41 years and the oldest patient was 78 years with a mean of 56.5. In this group there were 16 females and 27 males. This is a retrospective case study of 205 cases presenting with vertigo. The modality of treatment was Epley's manoeuvre in cases diagnosed with BPPV. The age of patients ranged from 41 to 78 years with mean of 56.5. The over all success rate was $96 \%$. All cases were instructed to report recurrence and were followed for 1 year.
\end{abstract}

Keywords Benign paroxysmal positional vertigo . Epley's manoeuvre - CSOM - Dix-Hallpike manoeuvre · Singular neurctomy $\cdot$ Vestibular neurectomy

\section{Introduction}

Benign paroxysmal positional vertigo (BPPV) is a common cause of vertigo. BPPV diagnosed in $20.9 \%$ of 205 cases reporting for vertigo in my study. BPPV is often misdiagnosed and over investigated by physicians, BPPV may occur concomitantly with other middle ear diseases such as CSOM with central perforation.

BPPV was first described by Barany in 1921 [1]. He also attributed BPPV with otolithic organs. In 1952 Dix and

K. K. Patangay $(\bowtie) \cdot R$. Ansari

Career Institute of Medical Sciences, Near IIM Road Ghaila,

Sahara City Homes, Lucknow 226020, India

e-mail: kpatangay@ rediffmail.com
Hallpike performed provocative positional testing named in their honour. In this article is posterior semicircular canal BPPV is included, although BPPV is also attributed to other semicircular canals which are less common.

Onset of BPPV is typically sudden. Many patients wake up noticing vertigo in BPPV. Patient often avoids positions triggering vertigo. Untreated symptoms of BPPV may extend for days to weeks, occasionally for months or years. In many cases symptoms resolve. Author has come across five which resolved on its own without any treatment. Such cases do not form a part of this study. After resolution of symptoms spontaneously over a period, symptoms may then recur. BPPV in severe cases may be associated with nausea and sometimes with vomitings. When BPPV is triggered, patients feel as though they are suddenly thrown into rolling spin, toppling towards the side of affected ear. Symptoms are violent and may last for 20-30 s [1].

\section{Materials and Method}

This study was carried out at Career Institute of Medical Sciences, Lucknow, India from Dec 2012 to Aug 2014. Thorough physical and ENT examination was carried out in all patients reporting with vertigo. Spontaneous nystagmus if any noted. Tests for cerebellar function in form of asynergia, asymetria, rebound and disdiadokinesis carried out to rule out cerebellar causes for vertigo.

Dix-Hallpike manoeuvre is the standard clinical test for BPPV. This test is performed by making the patient sit upright on a examination couch and the head is turned to one side at $45^{\circ}$ and then briskly taking head backwards with head hanging at $30^{\circ}$ over the back of the couch. Spectacles if any must be removed. Patient is told not to close eyes during the procedure and look into eyes of examiner. Wait for 
approximately $20-30 \mathrm{~s}$ for nystagmus to appear. If no nystagmus is noticed make the patients sit up. Now the same procedure is repeated with the head turned to the opposite side at $45^{\circ}$. If the Dix-Hallpike procedure does not evoke any nystagmus it is declared as negative. However if it does show nystagmus associated with vertigo it confirms diagnosis of BPPV. The classic BPPV of posterior SCC produces geotropic rotatory nystagmus associated with vertigo. The fast component of nystagmus is towards under most (affected) ear. Purely horizontal nystagmus indicates horizontal SCC involvement.

\section{Treatment}

Medical treatment in form of vestibular sedatives has no role in treatment of BPPV. Sometimes BPPV is self resolving condition. Definitive treatment is performing Epley's manoeuvre [2]. The manoeuvre consists of five positions.

Position 1: Sitting patient's head is turned towards the affected ear by $45^{\circ}$ and is rapidly brought downwards in head hanging position. Nystagmus appears after 20-30 s. This is called latent period. Keep the head in that position till the nystagmus disappears.

Position 2: Head is turned to the opposite side with the affected ear uppermost. Wait for 2 min.

Position 3: The whole body and head are now turned away from affected ear to a lateral recumbent position. Wait for $2 \mathrm{~min}$.

Position 4: Patient is brought to the sitting position with head turned away from affected side by $45^{\circ}$. Wait for 2 min.

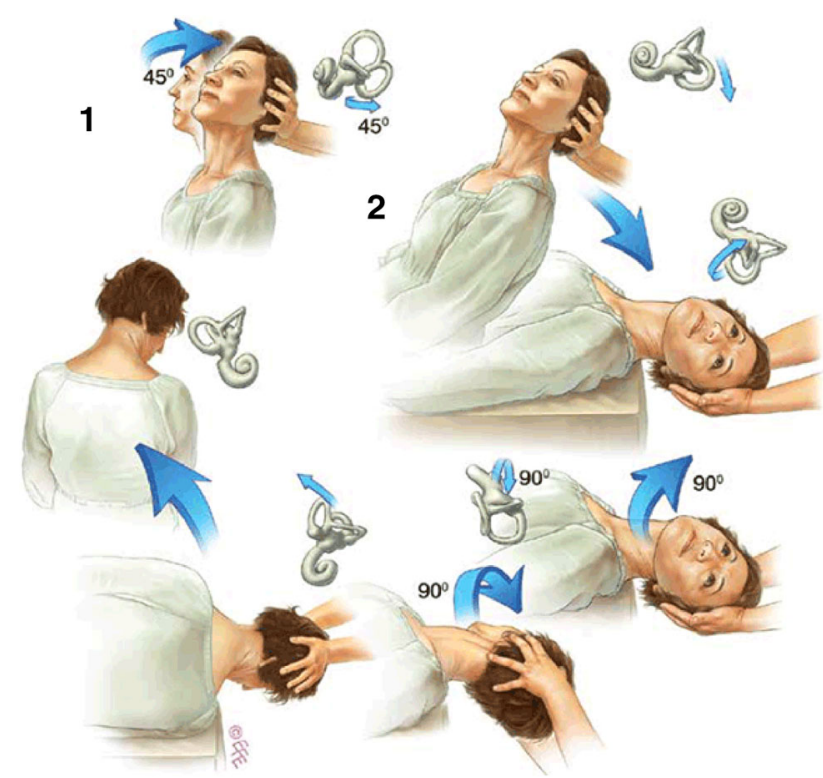

Fig. 1 The Epley's manoeuvre for treating left-side posterior semicircular BPPV
Position 5: With the patient still in sitting position bring his face forwards with chin $20^{\circ}$ downwards and wait for 2 min. Fig. 1:

Patient is now instructed not lie down for $12 \mathrm{~h}$ (Some authors recommend up to $48 \mathrm{~h}$ ). In addition patient is asked not to look up for at least a week.

In my study causes of BPPV is idiopathic. However other causes of BPPV can be head trauma, otitis media, vestibular neuritis, Meniere's disease, sudden sensorineural hearing loss etc [1].

\section{Discussion}

The diagnosis of BPPV is straight forward. History taking is the most important part in coming to provisional diagnosis. Confirmation of BPPV is always by performing Dix-Hallpike procedure. The exact aetiology of Idiopathic BPPV is vague. It is thought to be degenerative process in the vestibular organ. Otoconia from utricular macula get detached. These crystals of calcium carbonate float freely in endolymph of vestibule and get deposited in posterior SCC which get stimulated with change of position causing vertigo.

Epley's manoeuvre [3, 4] is nothing but repositioning detached particles of otoconia. This manoeuvre gives excellent results provided the procedure is properly carried out and patient follows strict instructions. Recurrences are known to occur. Repeat Epley's manoeuvre can be done again. In refractory cases obliteration of posterior SCC with bone dust has been described. Other operative procedures being Singular neurectomy which carries risk of sensorineural deafness and section of vestigular nerve supplying posterior SCC which carries post operative morbidity.

Semont's manoeuvre is another non invasive procedure. There is very little literature available regarding results in standard text books.

\section{Conclusion}

Results of our retrospective study showed that Epley's manoeuvre is very effective in treating patients with posterior SCC BPPV. Recurrences are known to occur but repeat Epley's manoeuvre gives relief in most of the cases. Medical treatment in form of vestibular sedatives have no role in management of BPPV. Surgery for Posterior SCC BPPV is invasive, requires specialised training and expensive. Epley's manoeuvre is single most mdality of treatment of BPPV [5].

\section{References}

1. Li JC et al (2014) Benign paroxysmal positional vertigo. Medscape, Aug 18 
2. Epley JM (1960) New dimensions of benign paroxysmal positional vertigo. Otolaryngol Head Neck Surg 88(5):599-605

3. Epley JM (1992) The canalith repositioning procedure for treatment of benign paroxysmal positional vertigo. Otolaryngol Head Neck Surg 107(3):399-404

4. Fung K, Hall SF (1996) Particle repositioning maneuver: effective treatment for benign paroxysmal positional vertigo. J Otolaryngol 25(4):243-248

5. Herdman SJ, Tusa RJ, Zee DS et al (1996) Single treatment approaches to benign paroxysmal positional vertigo. Arch Otolaryngol Head Neck Surg 119(4):450-454 